\title{
The Role of Mediators in Transforming and Translating Information Quality: A Case of Quality Assurance in a Norwegian Hospital Trust
}

\author{
Geir Inge Hausvik \\ University of Agder, Norway \\ geir.i.hausvik@uia.no
}

\author{
Devinder Thapa \\ University of Agder, Norway \\ devinder.thapa@uia.no
}

\author{
Bjørn Erik Munkvold \\ University of Agder, Norway \\ bjorn.e.munkvold@uia.no
}

\begin{abstract}
The existing literature on information quality (IQ) provides limited understanding of how roles influence $I Q$ in healthcare. The traditional way of understanding roles such as collectors, custodians, and consumers assumes that data are simply transformed into information and subsequently used by consumers. However, this does not explain how interpersonal communication influences IQ. In reality, the actors involved can actively change the quality of healthcare information through transformation, translation, or distortion. Latour's idea of intermediaries and mediators can be an appropriate lens for understanding these roles. Latour defined intermediaries as sociotechnical actors who simply transport information, whereas mediators can transform, translate, distort, and change the meaning of information. Following Latour's idea, we conducted a qualitative case study of quality assurance in a Norwegian healthcare organization. In doing so, we illustrated how IQ mediators can distort or create shared understanding of quality assurance information, which further influences enactment.
\end{abstract}

\section{Introduction}

The increasing adoption of electronic health record (EHR) systems in healthcare has become a critical area of research, since any compromise in the information quality (IQ) of EHR data can lead to dire consequences $[5,10,29]$. The ever-increasing amount of routinely collected data includes patient-level clinical data (e.g., documentation of clinical services delivered to patients, clinical findings, patient history, clinical orders, allergies, and laboratory results [43]) and administrative data (e.g., demographic data, socioeconomic data, financial data, and logistics data $[9,20])$.

EHR data are used by a multitude of users and their use is broadly categorized into primary and secondary [28]. Primary use of EHR data concerns supporting clinicians in decision-making at the point of care [18, 28], whereas secondary use of EHR data serves as a source of information for generating knowledge that may lead to improved healthcare systems and services. Examples of secondary uses include clinical audit and research, resource allocation, epidemiology, service planning, and performance monitoring $[18,28]$. The process of obtaining value from secondary use of data in healthcare organizations is characterized as ad hoc, with no standards in terms of empirical measures of core processes, and a lack of understanding of information needs. Further, this process is labor-intensive and timeconsuming, often conducted by manually exporting and manipulating data in third-party tools [14].

One noticeable impediment to the secondary use of EHR data is related to its quality [5], where high-quality information is claimed to be critical for effective and efficient management of healthcare systems [35]. IQ in an EHR context is referred to as information appropriate for healthcare interventions and processes, encompassing human, social, and technological elements of the context where information is produced, communicated, and used [6].

In existing IQ literature, information has often been treated as a product in which data is transformed into information through a manufacturing process [38]. The organizational roles involved in this process are data collectors, data custodians, and data consumers [24]. A similar approach has been applied to IQ research in the healthcare context [e.g., 34, 36]. However, this approach can be challenged, because it focuses on the technological effectiveness of EHR in producing quality information while neglecting the human aspects [30, 31]. As such, the traditional approach assumes a clearly delineated set of tasks for each role: collectors collect data, mediators maintain the computing resources of the information system (IS), and consumers access and use information products transformed from data by the IS. The nature of the human involvement in the process of transformation and interpersonal communication, however, remains unclear.

Interpersonal communication is argued to be a key characteristic that distinguishes use of IS in healthcare 
organizations from its use in other enterprises; whereas other enterprises use IS in transforming and communicating information, healthcare organizations additionally rely on person-to-person interaction $[1,30]$.

In reality, the actors can actively change the healthcare information through transformation, translation, or distortion. Latour's concepts of intermediaries and mediators from actor-network theory [23] serve as an appropriate lens for understanding these roles. Latour defined intermediaries as socio-technical actors who simply transport information, whereas mediators can transform, translate, distort, and change the meaning of information. Adopting Latour's perspective, we can argue that the traditional roles of human IQ actors are similar to those of intermediaries. This shift of focus from a technological view of understanding IQ to a more balanced socio-technical view, encompassing different users' views of IQ and interpersonal communication has been suggested as an avenue for contributions to IQ research $[30,32]$.

By acknowledging that interpersonal communication impacts the consumer view of IQ and, furthermore, the application of information, we argue that the roles are varied in nature and need to be understood as mediators. Drawing on Latour's concept of mediators from actor-network theory [23], we seek to answer the following research question: How does the role of mediators contribute to information quality in healthcare? This question is addressed in our study by analyzing data from a case of quality assurance in a Norwegian hospital trust.

The remainder of this paper is organized as follows. First, the theoretical underpinnings of the research are introduced, followed by presentation of the case. Then the research methodology is described, followed by case analysis, discussion, and conclusions.

\section{Theoretical background}

The theoretical concepts employed in this paper are IQ, intermediaries, and mediators. The following sections describe the relationships among these concepts.

\subsection{Information quality}

In the past three decades, numerous models and frameworks have tried to capture the concept of IQ. Common to these models are the entities of which they are constituted, often referred to as quality dimensions or quality elements. Examples of such entities include accuracy, reliability, timeliness, relevance, and completeness of information.
The most frequently adopted definitions of IQ are fitness for use [32] and fitness for purpose [12], where both definitions take an information consumer's point of view. Within the clinical specialist literature, IQ has been similarly defined as information appropriate for healthcare interventions and processes [6]. However, the majority of research in the EHR context has focused on specific dimensions of IQ (i.e., completeness, accuracy, comprehensiveness, and reliability [19]), without connecting this to any particular perspective on IQ.

The main differences between existing models from the general IQ literature, however, are their perspectives on IQ [3, 15]. Examples of such perspectives include hierarchical [42], ontological [40], semiotic [17], internal and external [11], evolutional [27], artifact and deliverable [26], product [41], and product and service [21]. Common to the existing views is the assumption that data is the input and information products are the outputs of a process performed by an IS [21, 33, 41]. Thus, IQ relates both to the features of the information product and to the features of its delivery process from the IS to the information user.

The literature refers to three distinct data processes in the life-cycle of information products: data production, data storage and maintenance, and data utilization [7]. Three distinct roles of human actors are involved in these processes, often referred to as the three Cs: data collectors, data custodians, and data consumers [7, 24, 34]. Data collectors (also referred to as data producers [21, 38] and data suppliers [2, 3, 41]) are actors providing initial input of organizational data to the IS [7, 24]. In an EHR context, data collectors include medical staff, nursing staff, and administrative staff [8]. Data custodians (also referred to as data manufacturers $[3,41]$ and data stewards [2]) are actors providing and managing computing resources for storing and processing data [21, 24, 38], a role often held by database administrators and computer scientists [8]. Finally, data consumers (also referred to as data users [25]) are organizational actors utilizing data for further integration, aggregation, presentation, and interpretation $[3,21,38]$, a role held by physicians, researchers, and managers within healthcare organizations [8].

Other roles have been suggested in the literature, including both generic roles (e.g., information product managers [41]) and context-specific roles (e.g., personal health information managers [34]), with the purpose of managing information processes and the resulting information products. However, existing research still treats the output of the IS as the final information product for the consumers, where the IS acts as a simple mediator between inputs and outputs [33]. 


\subsection{Intermediaries and mediators}

In actor-network theory, Latour [23] distinguishes between intermediaries and mediators. Intermediaries are defined as human or technological actors "transporting meaning or force without transformation" (p. 39), where defining the inputs of the actor is enough to define its outputs. Mediators, on the other hand, are human or technological actors that "transform, translate, distort, and modify the meaning or the elements they are supposed to carry" (p. 39).

The description of the information manufacturing process in existing IQ research, where an IS is considered as the sole mediator in transforming data inputs to information outputs, is limited in the healthcare context. In practice, human actors are also involved in transforming data into information. Furthermore, information outputs of the EHR are not always used directly; they may be communicated to other users. Such interpersonal communications are evident for both primary use of data (e.g., collaborative diagnosis and treatment assessment) and secondary use of data (e.g., organizational planning and decision-making) [1]. Thus, from Latour's perspective, viewing IS as the single most important mediator of information is insufficient; human actors communicating the information output of an IS to other humans are also important mediators. Moreover, this communication can also be facilitated by technology acting as an intermediary [13]. We argue that such human mediators affect IQ as perceived by information consumers, through transformation, translation, distortion, and modifications of the meaning of information.

\section{Case description}

Coastline Regional Hospital (CRH) (a pseudonym) is a large Norwegian public hospital providing specialist-level healthcare services to approximately 300,000 inhabitants, covering over 16,000 square kilometers of urban and rural areas. More than 7,000 employees work in different medical divisions, service departments, and administration throughout the region. Directors of medical divisions are responsible for specific disciplines, such as medicine, surgery, and psychiatry. The divisions comprise different departments supervised by department managers. Each department is subdivided into units led by unit managers. Some units are further divided into teams for purposes of division of labor. The psychiatry and addiction treatment division is one of six medical divisions within CRH; it consists of eight departments and has over 2,000 employees. In this study, we focus on secondary use of EHR data for quality assurance in this division.
The first version of the EHR was implemented in CRH in 1991 and contains electronic patient records for all patients attending the hospital after its implementation, including pre-1991 digitized paper records. The EHR consolidates converted data from several hospital mergers and legacy systems, and consists of structured data (e.g., diagnostic codes, hospital contact data, and demographics), semistructured data (e.g., XML-based forms), and unstructured data (e.g., journal documents). Journal documents are free-text medical narratives for which templates are selected by clinicians based upon the task. By March 2017, the EHR comprised about forty million journal documents related to 665,000 individual patients.

Data from the EHR is used for quality assurance at the division in two complementary ways: auditing unstructured data, and extracting structured data into a balanced scorecard (BSC). Since the EHR is designed for primary use of data (i.e., patient treatment at the point of care), functionalities for collecting multiple quality assurance measures and presenting the development of such measures over time are missing. Therefore, the division introduced the BSC in 2007 for collecting information for management purposes from different systems, including the EHR. The BSC is a standalone spreadsheet application that is updated every month by administrative advisors. In this process, source data are retrieved using built-in reports of the EHR and manually plotted in the BSC. The BSC visualizes periodical development of indicators from all departments in the division, as well as the degree of achievement of goals set by local, regional, or national government bodies.

For unstructured data, the only way of evaluating and assuring compliance with clinical guidelines is by performing medical journal audits. Such audits are performed at both department and unit levels in CRH. However, since auditing is labor-intensive and timeconsuming, it is performed at irregular intervals and with alternating focus. At the department level, quality advisors plan and organize the audit. The audits are performed by medical specialists, and the patient journals included in the audit are randomized. For each assessment, the results are plotted in an external dataprocessing tool in which data can be analyzed and visualized. Department audits are often followed by unit audits to refine the challenges and pinpoint where each challenge is rooted, allowing improvement interventions to target those units. However, no standards exist for collecting unit audit results, leading to the involvement of several different data-processing tools (e.g., surveying tools, spreadsheets, word processors, and paper-based audits). Department auditors tend to prefer a standard surveying tool, and 
unit auditors tend to prefer a spreadsheet application for structuring the findings, because of its functionalities, which include descriptive statistics and possibilities for visualizing the results.

When quality assurance data are collected, assessed, organized, analyzed, and visualized, the results are communicated to managers at various levels in the division. Then, the managers discuss the results and prioritize accordingly: decisions are taken, and responsibilities for actions are delegated. Finally, responsible individuals act upon the prioritized interventions. Such interventions always invoke some change in work processes and are often supported with training sessions. This final phase is crucial in terms of continuous quality assurance in the division, because the actual benefits of prioritized interventions cannot be realized without operational-level enactment.

\section{Research method}

Our research approach was interpretive [39], which is appropriate for the discovery of answers to our explorative research question. We conducted an interpretive case study at $\mathrm{CRH}$ in Norway by interviewing various stakeholders at different locations of CRH. The first author was involved in quality assurance at this hospital, which facilitated access to key stakeholders.

The sources of data included semi-structured interviews with employees, direct observations of quality assurance activities, collection of audit reports, spreadsheet templates used in data extraction, and minutes of meetings. The data were collected from all organizational levels in three different departments in the division, following the line of management from division level to department level, unit level, team level, and clinicians at the operational level.

Using snowball sampling, we identified relevant stakeholders in the line of management, such as administrative personnel, managers, and clinical personnel (e.g., nurses, psychiatrists, and psychologists). In total, thirty-one interviews were conducted during the period from September 2016 to June 2017. The average length of the interviews was sixty minutes. To further elaborate some questions, we exchanged follow-up emails with several interviewees. We confirmed participants' consent to record the interviews. All recorded interviews were transcribed and imported into NVivo 11 for further analysis.

We used thematic analysis to analyze the collected data [4]. The analysis started with open coding and categorization of the data. In the first round of coding, we identified all socio-technical actors involved in handling data and/or information in the process of quality assurance. In the second round of coding, we used Latour's concept of mediators in identifying events of transportation, transformation, translation, and distortion of information. Finally, we connected sociotechnical actors, identified in the first round, to events identified in second round. The categorization process was based on the iterative process of moving around data, concept, and categories, as specified by Klein and Myers [22] in their principles for evaluating interpretive field studies. Discussions with other researchers and practitioners were conducted throughout the study to ensure the validity of our interpretation. The different backgrounds of the authors, with one being involved in the quality assurance process at $\mathrm{CRH}$ and the others being outsiders, facilitated an in-depth and critical analysis of the research context.

\section{Case analysis}

The traditional roles in IQ, such as data collectors, data custodians, and data consumers, have been studied from a primary use perspective. However, our study is focused on secondary use of EHR data and the various roles, including the mediator role, associated with secondary use (see Figure 1). Our study shows the relevance of these roles in production, storage, maintenance, and utilization of data for quality assurance at the psychiatry division of CRH. In the subsequent sections, we describe these roles in detail.

\subsection{Collectors}

The quality assurance process at $\mathrm{CRH}$ relies on secondary use of EHR data. In this process, existing data are collected from the EHR, where the purpose is to assess the current quality of healthcare services and discover opportunities for improvements. Although both structured and unstructured EHR data are collected in this process, the method and actors involved are different.

5.1.1. Collecting structured data. Structured EHR data (for example bed days, re-admissions, and treatment waiting time, etc.) are collected using built-in EHR reports. The data collected from the EHR are entered into a data-processing tool in which data are organized and transformed into quality indicators (for example, average bed days, re-admission intensity, and average waiting time). This process of collecting structured data is performed by administrative staff at CRH both on a regular basis (e.g., monthly, as input for the BSC) and on an ad hoc basis. The most frequently used tool for collecting structured data is a standard spreadsheet application.

At CRH, data collectors emphasize correctness as an important quality dimension in the process of collecting 
structured data, as illustrated by one of the informants:

When I work with the balanced scorecard, my goal is that the data I collect must be as correct as possible. (Administrative consultant, Department level)

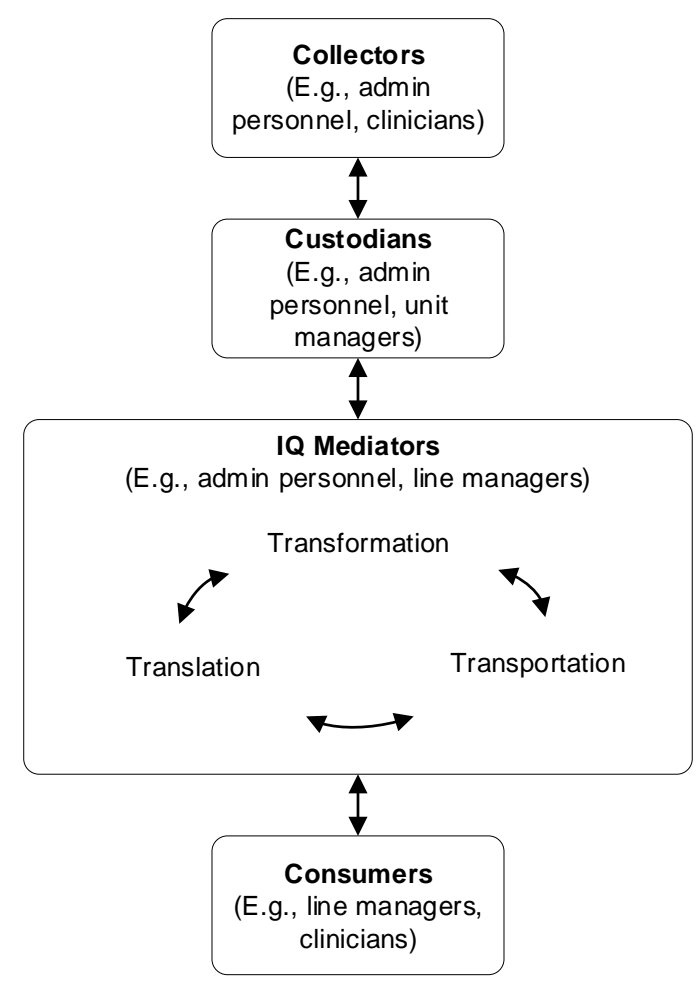

Figure 1. Roles of IQ mediators in quality assurance

5.1.2. Collecting unstructured data. The unstructured journal documents in the EHR contain narratives describing the services provided to patients, including clinical assessments. The only way of evaluating the level of compliance of services with the clinical guidelines at $\mathrm{CRH}$ is by performing medical audits. Since collection of such data involves assessments of the data content, collectors need medical competence:

Someone with medical knowledge must do it [collect the data], because it is not an exact science, where something is either present or absent. Also, people express themselves differently, and mostly in narratives. (Senior quality advisor, Division level)

EHR audits are performed at both department and unit levels at CRH. Findings at the department level often trigger unit-level audits to pinpoint challenges and target improvement interventions. In addition to medical experts, unit managers are often involved in data collection for unit-level audits.
After data has been collected from the EHR, collectors manually enter their assessments into a dataprocessing tool, where the purpose is to organize the data in preparation for further analysis. A range of different data-processing tools is used for collecting audit data, including a standard surveying tool, a standard spreadsheet application, an information processing tool, and even paper-based data collection. The tendency at CRH is to use less sophisticated tools at the unit level and more sophisticated tools at the division level.

At $\mathrm{CRH}$, data collectors of unstructured data emphasize objectivity as an important quality dimension, and several measures are taken to avoid biases. For example, randomization of patients was important to avoid biases in medical audits:

It was a randomized selection [of patients] ... where we evaluated how [clinicians] documented ... You need to read through many journals ... and if you select someone [patients] that you know, it might get really biased. So, you need the competence to perform randomized selections. (Medical advisor/psychologist, Division level)

\subsection{Custodians}

In the quality assurance process at $\mathrm{CRH}$, data custodians are involved both in the preparation for data collection and in the organization of the data collected. For example, before division audits, the data custodian provides instructions for how data collectors must collect the data. Furthermore, custodians customize the data-processing tool to secure a coherent collection and organize data to facilitate further analysis.

IT personnel are not involved as custodians in the quality assurance process at CRH. For division initiatives, such as department-level audits and data collection for the division-level BSC, the role of data custodians is prominent and held by division-level administrative staff. At unit levels, however, this role is less prominent and often intertwined with other roles. For department-/unit-level collection of structured data, administrative staff often hold the roles of both data collectors and custodians. For unstructured data at unit levels, unit managers often hold the role of data custodian, and sometimes also the role of data collector:

When [the audit] was being operationalized, I chiseled out some clear questions. I believe this became some sort of a standard that the other [units] copied. Then I collected [data] and analyzed it. (Unit manager)

The main IQ challenge in relation to data custodians at $\mathrm{CRH}$ is the consistency dimension. This is particularly 
evident at lower organizational levels at CRH, where unit managers hold the role of data custodian. In such cases, unit managers have a high degree of freedom in setting audit criteria and choosing data-processing tools for collecting, organizing, and storing data. This leads to inconsistencies in quality assurance data between units. A department-level advisor described this problem in the context of trying to collect all unit-level audit results from one department:

I made a somewhat standardized form for what they were supposed to evaluate, but it became obvious that they were evaluating far too much ... What I learned [is that] ... some things were [audited] consistently across all units. But additionally, some [units] included other [subjects] as well. The [audit results] cannot be compared, because some [units] included things that others didn't. What I learned was to provide a template next time. I assumed they'd all be evaluating the same things. (Quality advisor, Department level)

\subsection{Mediators}

According to Latour, transportation of information is performed not by mediators but by intermediaries [23]. Findings from the present study suggest, however, that the distinction between intermediaries and mediators is not clear-cut, and the roles are sometimes intertwined. Therefore, when referring to the mediator role, we need to distinguish among the actions taken, i.e., transportation, transformation, and translation.

Quality assurance data are not readily available for users at CRH but need to be collected from existing EHR data and stored in separate data-processing tools. Furthermore, information products are manufactured by human actors using functionalities of such tools, which in turn are communicated to accountable individuals for enactment. In this process, the role of mediators is vital for three distinct purposes: transformation, translation, and transportation of quality assurance information.

5.3.1. Transformation. The role of mediators involved in transformation at $\mathrm{CRH}$ is primarily concerned with transforming existing EHR data into quality assurance information. This is a highly socio-technical process that involves both human actors and data-processing technology, where mediators use the functionalities of data-processing tools to generate information products. For structured EHR data, the role of mediators is often held by administrative personnel, most frequently using a standard spreadsheet application as a data-processing tool. The role of mediators in the transformation of data into information is illustrated by a quality advisor at CRH:
We have an advisor - a number cruncher. It's basically what she does: making reports - numbers, percentages, monthly, weekly, and by unit managers' requests. She's that kind of a person $-a$ mediator, I would say ... Because of the enormous amount of data, you need such people working on this on a daily basis. (Quality advisor, Department level)

For unstructured data collected through divisionlevel audits, transformation of data is performed by division-level staff. This is often the same person who holds the custodian role. Transformation is done using the analytical and descriptive functionalities of the dataprocessing tool, followed by visualization of the findings in report format. At lower organizational levels, transformation is less systematic than at division level, where unit managers often hold the role of mediator. However, transformation of quality assurance information takes place at all levels in $\mathrm{CRH}$, as illustrated by a unit manager:

Data and numbers are being adjusted all the way down [the line of management], because so many considerations must be taken into account for the [operational level] - somebody may end up on sick leave if it is not presented properly. (Unit manager)

As indicated above, one of the reasons mediators transform the information product is related to the information consumers' expectations of the information. Thus, mediators seem to be concerned with changing IQ dimensions according to consumers' perceptions when transforming information products. One mediator frequently mentioned that a challenge leading to transformation is related to the granularity dimension of IQ. Often, information products consist of aggregated data, without the possibility of identifying findings at unit levels. This is illustrated by one informant, who stated why department-level audit reports were insufficient, resulting in a need to transform department-level results into unit-level results:

Yes, [conducting unit-level audits] was of paramount importance. It doesn't have the same effect when division management performs audits ... because they [the line management] must own it. They must see it themselves ... They won't relate to this unless it gets broken down to their units. They don't need it and don't know how to use it. So, for them to take it seriously, we need to get it broken down to their unit. (Assistant department manager) 
5.3.2. Transportation and translation. After production, quality assurance information products are communicated within CRH. In this process, mediators transport and/or translate the information products to relevant actors. At $\mathrm{CRH}$, the line of management is prominent in the flow of information, making managers at all levels mediators of information to successive management levels. In terms of transportation, information products are simply transported from the mediator to information consumers. Transportation is often facilitated by technology (e.g., email), as illustrated by the words of one department manager:

Then [administrative staff] sends the balanced scorecard to me, and I forward it to my unit managers: "And here are the results for February". The number of referrals, number of rejections, number of patients not attending [appointments], waiting times, deadline violations, etc., etc. (Department manager)

Since information sometimes needs to travel through multiple mediators at different organizational levels before reaching end-users, simple transportation may be challenging. The main challenge in transportation at $\mathrm{CRH}$ is related to distortion due to overwhelming amounts of information competing for consumers' attention:

We're flooded by emails and reports. There are no limits to how much we receive. If my clinicians were supposed to read all of it ... But the [managers] above me just pass everything on ... so they can say "yes, but you've [already] got that." (Unit manager)

Such distortions can further disrupt the transportation of information between consumers at various organizational levels, as explained by a quality advisor:

We didn't reach the individual clinician. That is, the improvement information about how to do things differently because it's important to patients. It never reached them ... you know, reaching the lowest level-it stopped at the first and second levels [in the line of management]. And I believe this is the core of the challenge ... All levels must want it, and the lowest level must recognize its importance. (Quality advisor, Division level)

One of the characteristics of transportation is that the IQ of the information product remains unchanged when communicated from one individual to another. In the quality assurance process at $\mathrm{CRH}$, informants emphasized that transportation was insufficient, since departmental audit results and BSCs were intended for management purposes, not for a general audience. This is illustrated by a department manager's explanation of why he chose to suspend transportation of an audit report to clinicians:

No - they are allergic to this. It's the amount. It's graphs and tables ... It has colors and everything. They [the clinicians] want it to be explicit. This is too much - it's [intended] for people like me. (Department manager)

Nevertheless, for enactment to happen, it is important that quality assurance information is communicated to and understood by clinicians. Two IQ dimensions in particular can hamper shared understanding in simple transportation; there is a possibility that information consumers fail to understand the content and that they fail to see its relevance. This in turn leads to failure of enactment:

When someone states that you've got a problem, and you don't understand the problem - then, how can you do anything with it? ... Then it definitely doesn't apply to you, and you've got social loafing: all of a sudden, it doesn't apply to anyone. (Medical advisor/psychologist, Division level)

Thus, mediators need to translate the information product to consumers in order to reach a shared understanding. This is illustrated by a clinician's evaluation of the usefulness of an audit report:

I think that [the audit report] is boring and hard to understand. I don't understand everything. It's so much easier when you have a person in front of you that you can talk to and ask if you are wondering about something. (Clinician)

The main aim for mediators in translation is to reach a shared understanding of the information and its implications. Managers at all levels are crucial when acting as mediators in the process of translation, as explained by the division director:

How the information flow is being handled at all the [organizational] levels is a critical factor. This has to do partly with how we provide the information, and partly with the content of the information itself ... You may say that it's critical at all management levels, when you bring information about a phenomenon from one person to another person - to another person's brain. (Division director)

Translation is also associated with distortion, since shared understanding is required in sequences and across organizational levels, as the division director stated: 
It's like that whispering game, where people are whispering something to the next person. And, eventually, you see whether the information ends up like it started. It's precisely the same - it's a whispering game. And it's absolutely critical for the task or phenomenon, and particularly critical if it concerns an agreed-upon change. (Division director)

In translation, mediators change the IQ of the information product to reach a shared understanding. In particular, mediators aim to increase its understandability and relevance for the information consumer.

\subsection{Consumers}

Consumers are usually considered as end-users of information (e.g., clinicians). However, this study shows that consumers also exist at various organizational levels prior to the end-users. For example, managers can be consumers, but they are at the same time mediators of information to subsequent consumers in the line of management.

\subsection{Summary of case analysis}

Our case analysis documents the existence of the traditional roles of data collectors, data custodians, and data consumers. These roles, however, are static in nature, and they do not explain how actors modify IQ in the quality assurance process. The case analysis revealed how different mediators transform both structured and unstructured EHR data into quality assurance information by embedding the IQ dimensions that they believe are important to information consumers. Transformation of data is dependent on the IQ dimensions embedded by data collectors (e.g., correctness and objectivity) and by data custodians (e.g., consistency). In some cases, where information lacks important IQ dimensions (e.g., granularity), the process of transformation of EHR data needs to be reiterated.

After its transformation, information is communicated to consumers within the organization by transportation or translation. Since transportation preserves the IQ of the information product, actors often fail to understand or see the relevance of the information. These important IQ dimensions are addressed when mediators translate the information and thereby reach a shared understanding.

\section{Discussion}

From the existing information manufacturing perspective, EHR data are collected by data collectors, maintained by data custodians, and transformed into information and made available by an IS for data consumers [34, 36, 38]. IQ is assessed as high if the information product is fit for use [32] or appropriate for healthcare interventions [6], and there is an assumption that consumers will act on given information if IQ is maintained. However, the caveat in the existing approach is the inability to differentiate between IQ in primary and secondary use of EHR data. As identified in this study, secondary use of EHR data for quality assurance is a highly socio-technical process. Compared to primary use of data, human actors are more involved in the process of transforming data into information in secondary use. In this case, IT personnel were not involved in the process of secondary use. Furthermore, the information products resulting from this process were communicated and transformed throughout the organization before reaching end-users. For example, since quality assurance information is not readily available from the EHR, mediators heuristically transform EHR data into quality assurance information by using various data-processing tools. The mediator's role in transformation, translation, and transportation is rarely discussed in the existing literature. In this paper, we contribute by revealing how the human interaction in secondary use of EHR data can change its IQ, which in turn can affect quality assurance.

To reveal the transformation and translation process, we draw upon Latour's concepts of mediators and intermediaries [23]. The traditional approach of defining roles focuses mainly on the intermediaries, who simply transform and transport the information to end-users, whereas we argue that it is necessary to identify the mediators, who not only transform and transport but also translate the meaning. Our study shows that mediators also engage in communicative actions in providing information to relevant consumers. Such provision of information by mediators is identified as transportation and translation. In transportation, quality assurance information is simply transported from one actor to another, without any modifications of the information product or the IQ. In translation, however, mediators actively translate the information with the aim of achieving a shared understanding between the mediator and the consumer. A key finding of this study is that translation should focus on enhancing the IQ dimensions of understandability and relevance when communicating to consumers.

In the traditional view of IQ, the quality of transformation and transportation of information products from IS to consumers are characterized in terms of service quality [21]. However, our analysis shows that mere transformation and transportation of information is insufficient for enactment. In fact, the mediators need to translate the information product to 
reach a shared understanding, which in turn may lead to enactment. This finding is in line with the work of Eppler [13], who introduced communication quality as the quality of interpersonal communication. Furthermore, Lillrank [26] introduced a bipolar view of IQ, distinguishing between quality of information-asan-artifact (equivalent to information as a product) and information-as-a-deliverable. The latter views deliverables as negotiations between producers and receivers, where good IQ is defined as shared understanding. This view is consistent with the findings of this study and with our notion of mediators as translators.

In existing research, data collectors include medical and administrative staff, data custodians include database administrators and computer scientists, and data consumers include physicians and managers [8]. As our case illustrates, this classification of professionals does not describe the nature of secondary use of data. For example, IT professionals were not involved in this process at all; administrative staff, and even managers, held the role of data custodians. Furthermore, individuals could hold several roles in the process, making distinctions between roles sometimes hard to identify.

Moreover, we found that when information is simply transported from producer to consumer, it may not lead to enactment. This could be because of distortions in transportation, lack of user understanding, or lack of relevance in a user context. In other terms, we can say that users were unable to perceive the action possibilities, or affordances, of the information. There are a few examples of studies that use the theory of affordances as analytical lens [16,37], but the role of IQ is rarely mentioned in these studies. Combining the concepts of mediators and affordances can be a productive endeavor for future research.

This study has a number of implications for practice, such as the role of mediators in creating shared understanding and demonstrating the relevance of information to consumers. Furthermore, we have identified the crucial role of mediators in translating quality assurance information as a prerequisite for enactment. In particular, in the case of secondary use of EHR data, we identified line managers as key actors holding the role of mediators, whereas IT personnel play a less important role.

\section{Conclusion}

Existing literature on IQ focuses on the roles involved in primary use of EHR data, without addressing the interpersonal communication characteristic of secondary use. In this paper, we pointed out the knowledge gap in existing IQ research in terms of understanding the role of mediators in transforming, translating, and transporting information in secondary use of EHR data. In doing so, we formulated the research question: how does the role of mediators contribute to information quality in healthcare? To answer the question, we presented a case study and applied Latour's notion of mediators to make sense of our data. Our findings show how mediators can influence the quality assurance process in a healthcare context through changing IQ. Finally, we stated the implications for research and practice and proposed future research avenues.

\section{References}

[1] Avison, D. and T. Young, "Time to Rethink Health Care and ICT?", Communications of the ACM, 2007, pp. 69-74.

[2] Baškarada, S. and A. Koronios, "A Critical Success Factor Framework for Information Quality Management", Information Systems Management, 2014, pp. 276-295.

[3] Batini, C., et al., "Methodologies for Data Quality Assessment and Improvement", ACM Computing Surveys, 2009, pp. 16:1-16:52.

[4] Braun, V. and V. Clarke, "Using Thematic Analysis in Psychology", Qualitative Research in Psychology, 2006, pp. 77-101.

[5] Byrd, J.B., et al., "Data Quality of an Electronic Health Record Tool to Support VA Cardiac Catheterization Laboratory Quality Improvement: The VA Clinical Assessment, Reporting, and Tracking System for Cath Labs (CART) Program", American Heart Journal, 2013, pp. 434440.

[6] Cabitza, F. and C. Batini, "Information Quality in Healthcare" in Data and Information Quality: Dimensions, Principles and Techniques, C. Batini and M. Scannapieca (eds.), Springer, London, 2016, pp. 403-419.

[7] Cao, L. and H. Zhu, "Normal Accidents: Data Quality Problems in ERP-Enabled Manufacturing", ACM Journal of Data and Information Quality, 2013, pp. 1-26.

[8] Cruz-Correia, R.J., et al., "Data Quality and Integration Issues in Electronic Health Records" in Information Discovery on Electronic Health Records, V. Hristidis (ed.), Chapman \& Hall / CRC, 2009.

[9] Davis, N. and M. LaCour, Health Information Technology, Elsevier Saunders, St. Louis, MO, 2014.

[10] DesRoches, C.M., et al., "Electronic Health Records in Ambulatory Care - A National Survey of Physicians", New England Journal of Medicine, 2008, pp. 50-60.

[11] Devilliers, R. and R. Jeansoulin, "Spatial Data Quality: Concepts" in Fundamentals of Spatial Data Quality, R. Devilliers and R. Jeansoulin (eds.), ISTE Ltd, London, 2006.

[12] Embury, S.M. and P. Missier, "Forget Dimensions: Define Your Information Quality Using Quality View Patterns" in The Philosophy of Information Quality, L. Floridi and P. Illari (eds.), Springer, New York, 2014. 
[13] Eppler, M.J., Managing Information Quality: Increasing the Value of Information in Knowledge-Intensive Products and Processes, Springer, Heidelberg, 2006.

[14] Foshay, N. and C. Kuziemsky, "Towards an Implementation Framework for Business Intelligence in Healthcare", International Journal of Information Management, 2014, pp. 20-27.

[15] Ge, M. and M. Helfert, “A Review of Information Quality Research - Develop a Research Agenda" in Proceedings of the 12th International Conference on Information Quality (ICIQ), Cambridge, USA, 2007.

[16] Hausvik, G.I. and D. Thapa, "'What You See is Not What You Get' - Challenges in Actualization of EHR Affordances" in Proceedings of the 38th International Conference on Information Systems (ICIS), Seoul, South Korea, 2017.

[17] Helfert, M., "Managing and Measuring Data Quality in Data Warehousing" in Proceedings of the World Multiconference on Systemics, Cybernetics and Informatics, Orlando, USA, 2001.

[18] Hripcsak, G., et al., "Health Data Use, Stewardship, and Governance: Ongoing Gaps and Challenges: A Report from AMIA's 2012 Health Policy Meeting", Journal of the American Medical Informatics Association, 2014, pp. 204 211.

[19] Häyrinen, K., K. Saranto, and P. Nykänen, "Definition, Structure, Content, Use and Impacts of Electronic Health Records: A Review of the Research Literature", International Journal of Medical Informatics, 2008, pp. 291-304.

[20] Jensen, P.B., L.J. Jensen, and S. Brunak, "Mining Electronic Health Records: Towards Better Research Applications and Clinical Care", Nature Reviews Genetics, 2012, pp. 395-405.

[21] Kahn, B.K., D.M. Strong, and R.Y. Wang, "Information Quality Benchmarks: Product and Service Performance", Communications of the ACM, 2002, pp. 184-192.

[22] Klein, H.K. and M.D. Myers, "A Set of Principles for Conducting and Evaluating Interpretive Field Studies in Information Systems", MIS Quarterly, 1999, pp. 67-93.

[23] Latour, B., Reassembling the Social: An Introduction to Actor-Network-Theory, Oxford University Press, Oxford, UK, 2005.

[24] Lee, Y.W., "Crafting Rules: Context-Reflective Data Quality Problem Solving", Journal of Management Information Systems, 2003, pp. 93-119.

[25] Lee, Y.W., et al., "AIMQ: A Methodology for Information Quality Assessment", Information \& Management, 2002, pp. 133-146.

[26] Lillrank, P., “The Quality of Information", International Journal of Quality \& Reliability Management, 2003, pp. 691703.

[27] Liu, L. and L.N. Chi, "Evolutional Data Quality: A Theory-Specific View" in Proceedings of the 7th International Conference on Information Quality (ICIQ), Cambridge, USA, 2002.
[28] Mann, R. and J. Williams, "Standards in Medical Record Keeping”, Clinical Medicine, 2003, pp. 329-332.

[29] Meidani, Z., et al., "Organization's Quality Maturity as a Vehicle for EHR Success", Journal of Medical Systems, 2012, pp. 1229-1234.

[30] Mettler, T., P. Rohner, and L. Baacke, "Improving Data Quality of Health Information Systems: A Holistic DesignOriented Approach" in Proceedings of the 16th European Conference on Information Systems (ECIS), Galway, Ireland, 2008.

[31] Mohammed, S.A. and M.M. Yusof, "Towards an Evaluation Framework for Information Quality Management (IQM) Practices for Health Information Systems - Evaluation Criteria for Effective IQM Practices", Journal of Evaluation in Clinical Practice, 2013, pp. 379-387.

[32] Neely, M.P. and J.S. Cook, "Fifteen Years of Data and Information Quality Literature: Developing a Research Agenda for Accounting", Journal of Information Systems, 2011, pp. 79-108.

[33] Orr, K., "Data Quality and Systems", Communications of the ACM, 1998, pp. 66-71.

[34] Osesina, O.I., M.-A. Carton-Mizeracki, and J. Talburt, “A Patient-Oriented Approach to Comprehensive Health Information Quality Improvement" in Proceedings of the 16th International Conference on Information Quality (ICIQ), Adelaide, Australia, 2011.

[35] Richards, H. and N. White, "Ensuring the Quality of Health Information: The Canadian Experience" in Handbook of Data Quality: Research and Practice, S. Sadiq (ed.), Springer Heidelberg, Berlin, 2013.

[36] Sachdeva, S. and S. Bhalla, "Semantic Interoperability in Standardized Electronic Health Record Databases", ACM Journal of Data and Information Quality, 2012, pp. 1-37.

[37] Strong, D.M., et al., "A Theory of Organization-EHR Affordance Actualization", Journal of the Association for Information Systems, 2014, pp. 53-85.

[38] Strong, D.M., Y.W. Lee, and R.Y. Wang, "Data Quality in Context", Communications of the ACM, 1997, pp. 103-110.

[39] Walsham, G., "Doing Interpretive Research", European Journal of Information Systems, 2006, pp. 320-330.

[40] Wand, Y. and R.Y. Wang, "Anchoring Data Quality Dimensions in Ontological Foundations", Communications of the ACM, 1996, pp. 86-95.

[41] Wang, R.Y., et al., "Manage Your Information as a Product", Sloan Management Review, 1998, pp. 95-105.

[42] Wang, R.Y. and D.M. Strong, "Beyond Accuracy: What Data Quality Means to Data Consumers", Journal of Management Information Systems, 1996, pp. 5-33.

[43] Ward, M.J., K.A. Marsolo, and C.M. Froehle, "Applications of Business Analytics in Healthcare", Business Horizons, 2014, pp. 571-582. 